\title{
PIETRO VERRI ET JEAN-BAPTISTE SAY: VALEUR, MONNAIE ET LOI DES DÉBOUCHÉS
}

\author{
ANDRÉ TIRAN (*)
}

RÉSUMÉ. - Nous voulons tenter ici de déterminer quelle influence la lecture de Verri a pu exercer sur Say. Faut-il limiter cette influence à ce que Say lui même indique dans une note du Traité, à propos du commerce, ou bien faut-il donner une place toute autre et bien plus fondamentale dans la constitution de sa vision théorique générale? Si celleci n'a pas été relevée jusqu'ici, cela tient peut-être à une attention insuffisante vis-à-vis des auteurs italiens qui, exception faite de quelques auteurs comme Beccaria et Galiani, sont peu cités par les économistes spécialistes de l'histoire de la pensée économique en France mais aussi ailleurs.

Nous verrons que contre Smith J.-B. Say reprend à Pietro Verri la conception de la valeur-utilité, et contre les physiocrates (et ce qu'il reste de physiocratique chez Smith) celle de la production entendue comme modification et non pas création de matière. Toutefois il reprend chez Smith toute la place centrale que celui-ci accorde à la production et à l'échange de valeur pour valeur et opposition aux faiseurs de système.

Le comte Pietro Verri est aux yeux de J.-B. Say avec Turgot, l'économiste le plus important qui précède Adam Smith au XVIII siècle en Italie. Dans le Discours Préliminaire de la cinquième édition du Traité (1826), Say souligne très fortement l'importance des économistes italiens. Il écrit: "Le Comte de Verri compatriote et ami de Beccaria, et digne de l'être, à la fois grand administrateur et bon écrivain, dans ses Meditazioni sull'economia politica, publiées en 1771, s'est approché plus que personne avant Smith, des véritables lois qui dirigent la production et la consommation des richesses".

$* * *$

ABSTRACT. - Pietro Verri and Jean-Baptiste Say: value, money and the law of markets.

The aim of this essay is to determine what influence Verri may have had on JeanBaptiste Say. Should we limit Verri's influence to what Say himself acknowledges in a footnote of the Traité concerning the value of goods, or should we recognize for Verri another and more fundamental role in the formation of Say's general theoretical frame-

(*) UMR CNRS triangle, Université de Lyon, France. 
work? If this question has not been raised so far, this may be due to the insufficient attention so far paid in France, but also elsewhere, to the Italian economists of the eighteenth century, except for authors such as Beccaria and Galiani. As we shall see in this essay, Jean-Baptiste Say takes up, against Adam Smith, Verri's conception of utilityvalue, while against the Physiocrats (and also against what remains Physiocratic in Smith) Say maintains that production is a transformation, not a creation, of matter. At the same time, Say derives from Smith the central importance assigned to the production and exchange of values for values, and the opposition against system builders.

In the eyes of Jean-Baptiste Say, Pietro Verri is the most important eighteenth century economist before Adam Smith. In the Discours préliminaire to the $5^{\text {th }}$ edition of the Traité (1826), Say strongly emphasizes the importance of Italian economists. As he writes there: "Count Verri, compatriot and friend of Beccaria, and both a good writer and a great administrator, in his Meditazioni sull'economia politica, published in 1771, approached more than anyone else before Smith the real laws that govern the production and consumption of wealth".

J.A. Schumpeter dans son Histoire de l'Analyse Economique présente Say comme le continuateur de Turgot et de Cantillon. Il ajoute que ces derniers sont ceux qui ont probablement le plus marqué son analyse de. ${ }^{1}$ Si cette appréciation ne nous parait pas discutable en terme de filiation, l'influence que Schumpeter leurs attribue doit être nuancée au profit du comte Pietro Verri qui est celui qui a le plus influencé la vision de J.-B. Say après Smith. Toutefois Schumpeter cite Verri dans la chaîne qui conduit de Galiani à Walras en passant par Say. ${ }^{4}$

1 Il est vrai également que J.A. Schumpeter à propos de la théorie de la valeurutilité écrit: "Même après 1776, cette théorie prévalut sur le Continent et il y a une ligne continue de développement de Galiani à J.-B. Say: Quesnay, Beccaria, Turgot, Verri, Condillac et bien d'autres de moindre renom contribuèrent à la fonder de plus en plus solidement". Histoire de l'Analyse Economique, vol. I, pp. 421-422 et vol. II, p. 160.

2 Concernant le Comte Pietro Verri voir F. Venturi, «Le 'Meditazioni sulla economia politica' di Pietro Verri. Edizioni, echi e discussioni», pp. 530-593 in Rivista Storica Italiana, Anno XC, fascicolo III, 1978.

3 Nous utilisons ce terme dans le sens que lui donne Schumpeter dans son Histoire de l'Analyse Economique, comme vision fondamentale et créatrice.

4 J.A. Schumpeter, Histoire de l'Analyse économique, vol. II, p.159: "L'œuvre de Say a des origines purement françaises si nous considérons Cantillon comme un économiste français. C'est la tradition Cantillon - Turgot qu'il continue et à partir de laquelle il aurait pu développer quoiqu'il ait pu faire en réalité tous les traits principaux de son analyse, y compris d'ailleurs son schéma systématique et son entrepreneur. Le plus important de 
Nous voulons ici déterminer quelle influence la lecture de Verri a pu exercer sur Say. Faut-il limiter cette influence à ce que Say lui même indique dans une note du Traité, à propos du commerce, ou bien faut-il donner une place toute autre et bien plus fondamentale dans la constitution de sa vision théorique générale? Si celle-ci n'a pas été relevée jusqu'ici, cela tient peut-être à une attention insuffisante vis-à-vis des auteurs italiens qui, exception faite de quelques auteurs comme Beccaria et Galiani, sont peu cités par les économistes spécialistes de l'histoire de la pensée économique en France mais aussi ailleurs.5

Nous allons confronter l'analyse de Verri et celle de Say. Nous verrons tout d'abord que Verri, à partir de la théorie de la valeur utilité-rareté combinée à l'analyse selon laquelle l'activité humaine n'est jamais une création de matière mais seulement une transformation de matière va:

1- Caractériser toutes les activité des hommes de productives (celles des membres de l'Etat excepté) et désigner par là ce qu'est la source de la richesse et donc la puissance d'un Etat.

2- Formuler les lois générales des rapports entre production, consommation et croissance.

3- Fonder la cohérence d'ensemble de l'édifice sur la théorie de la valeur en y intégrant la monnaie. La monnaie est désignée comme «marchandise universelle», possédant les mêmes attributs que toutes les autres marchandises et soumise aux mêmes lois, donc échappant au Prince. Cette conception permet de passer de l'idée particulière de valeur propre à deux participants à l'échange, à une idée générale de la valeur s’imposant à tous.

ces éléments est en vérité sa contribution à l'économie analytique et sa conception de l'équilibre économique bien qu'elle soit formulée de façon vague et imparfaite. L'œuvre de Say est le maillon le plus important de la chaîne qui nous conduit de Cantillon et Turgot à Walras". C'est précisément cette présentation de J.A. Schumpeter qui nous semble devoir être remise en cause, en particulier les "origines purement françaises".

5 Thomas Guggenheim dans son analyse des théories monétaires pré-classiques indique que faute d'une connaissance de la langue suffisante il n'abordera pas les auteurs italiens ce qui est un problème scientifique majeur compte tenur du niveau de la contribution italienne. T. Guggenheim, Les théories monétaires pré-classiques, Genève, Librairie Droz, 1978, p. 10. Relevons par ailleurs que la dernière édition fraçaise de l'Encyclopaedia Universalis ne consacre pas une ligne à Verri, pas plus que la précédente qui ne le cite que dans l'article sur Beccaria. 
4- Développer sa visée générale qui reste celle du conseiller du Prince mais qui sape en même temps les positions du Prince en ceci qu'elle donne un statut à la monnaie et aux activités économiques qui les émancipent largement du politique. Contre Smith Say reprend à Pietro Verri la conception de la valeur-utilité, et contre les physiocrates (et ce qu'il reste de physiocratique chez Smith) celle de la production entendue comme modification et non pas création de matière.

Tout ce travail de déconstruction/reconstruction lui permet de:

1- Caractériser toutes les activités humaines de productives y compris celle des fonctionnaires de l'Etat (sauf dans la première édition il suit la position de Verri).

2- D'abolir la distinction de Smith entre productif et improductif et d'introduire la notion de produits matériels et immatériels.

3- De fonder les lois de la répartition des revenus entre les différents services productifs en cohérence avec l'ensemble de la production.

4- La monnaie ne remplit que la seule fonction de moyen de circulation. Ce qui est premier c'est la production, celle-ci intègre la monnaie comme marchandise.

5- Il se sépare de Verri en achevant la rupture totale avec le politique, seul l'individu a en main son propre salut qui passe par la connaissance des lois de l'économie politique.

6- Il formule la loi des débouchés dans l'ordre même où Verri aborde la question.

\section{L'ANAlyse De Pietro VerRi}

\subsection{Une influence reconnue}

Le comte Pietro Verri est aux yeux de J.-B. Say ${ }^{6}$ avec Turgot, l'économiste le plus important qui précède Adam Smith au XVIII siècle en Italie. Dans le Discours Préliminaire de la cinquième édition du Traité (1826), Say souligne très fortement l'importance des économistes

6 J.A. Schumpeter porte un jugement identique, Histoire de l'analyse Economique, vol. I, pp. 253-254: "Le Comte Pietro Verri (1728-1797), qui était un agent de l'administration autrichienne de Milan et non pas un enseignant, doit être inclus dans toute la liste des plus grands économistes". 
italiens. Il écrit: «Le Comte de Verri compatriote et ami de Beccaria, et digne de l'être, à la fois grand administrateur et bon écrivain, dans ses Meditazioni sull'economia politica, publiées en 1771, s'est approché plus que personne avant Smith, des véritables lois qui dirigent la production et la consommation des richesses». ${ }^{7}$

Dans la première édition du Traité (1803) Verri est un des rares, avec Smith, envers lequel Say se reconnaisse une dette. Il le fait dans le chapitre qui précède celui sur la loi des débouchés à propos du commerce: «Le Comte Verri est, à ma connaissance, le premier qui ait dit en quoi consistait le principe et le fondement du commerce. Jusqu'à lui et depuis, on a sans cesse répété que le commerce était un échange de l'excédent de denrées dont chaque peuple pouvait disposer. On a pris le moyen pour le principe. Le Comte de Verri a dit en 1772: 'Le commerce n'est réellement autre chose que le transport des marchandises d'un lieu à un autre'. ${ }^{8}$ Dans son Histoire Abrégée de la Pensée Economique il écrit: Verri est un des esprits les plus judicieux qui aient écrit sur l'économie politique. Il voyait mieux le fond des choses que les économistes. Beccaria et lui étaient compatriotes et amis».?

S'il est un des très rare économistes à citer les italiens, il le doit en partie à l'éducation que son père lui a fait donner lorsque la famille habitait à Lyon..$^{10}$ En reprenant les passages où J.-B. Say cite le Comte Pietro Verri on constate que ce sont toujours des points sur lesquels il

7 Say, Traité d'Economie Politique (TEP), 5ème édition, tome I, p. lvj, Paris, 1826. Il écrit ailleurs dans cette même partie: "[d]ès le 16e siècle, Botero s'était occupé à chercher les véritables sources de la prospérité publique. En 1613 Antonio Serra fit un traité dans lequel il avait signalé le pouvoir productif de l'industrie", p. xli.

8 Say, Traité d'Economie Politique, 1ère édition, Paris, Crapelet, 1803, p. 147. Nous avons volontairement ici utilisé la première édition de 1803 en particulier sur la loi de la valeur et sur celle des débouchés afin de cerner ce qu'avait pu être l'influence initiale.

9 Say, Cours Complet d'Economie Politique, 1ère édition, Paris, Rapilly, 1828-29, tome VI, Histoire abrégée, p. 395. En note, J.-B. Say ajoute: "On en peut dire autant de l'observation suivante du même auteur: 'L'argent lui-même est une chose, un métal, dont la valeur est représentée par tout ce qu'on donne en échange pour l'avoir. La propriété de représenter la valeur, est commune à toutes les marchandises. Cette définition ne convient donc pas exclusivement à l'argent'». P. Verri, Meditazioni sulla economia politica, Ch. 2.

10 George Michel, dans le Journal des Economistes, 5e série, T. XXXIV, 1898, Paris, Guillaumin, p. 171, cite ce passage du début d'autobiographie de J.-B. Say: "On nous enseignait l'bistoire, telle qu'on la trouvait dans les livres de cette époque, c'est à dire une fable convenue, on nous enseignait la grammaire, la langue italienne assez bien, et le 
se reconnait une dette. Il y a tout d'abord cette note qui figure dans toutes les éditions du Traité concernant la définition du commerce comme «le transport d'un produit d'un lieu à un autre». Cette définition peut nous paraitre banale, voire triviale, mais dans la mesure où Say la souligne dès le début du Traité et au cours des cinq éditions qui suivront c'est qu'il lui accorde une certaine importance. La question est donc de savoir quel est l'enjeu de cette distinction? S'agit-il simplement de prouver que le commerce est aussi une activité productive, ou bien faut-il y voir un enjeu plus important par rapport à l'ensemble de la construction théorique de J.-B. Say?

Say situe l'apport de Verri comme le maillon de la chaîne qui de Quesnay conduit à Smith. Il précise que c'est Verri qui a dit que la reproduction n'était autre chose qu'une reproduction de valeur et que la valeur était la richesse, Smith opère le lien entre ces idées là.

\subsection{Production, richesses et dynamique des besoins ${ }^{11}$}

L'objet de l'économie politique pour Pietro Verri est différent de ce qu'il deviendra pour A. Smith et J.-B. Say. Pour lui il s'agit de dire par quels moyens l'économie politique bien dirigée augmentera la puissance de l'Etat. ${ }^{12}$ Nous sommes dans l'optique du conseiller du Prince, il n'y a pas encore la présence indépendante du peuple qui commande en grande partie toute la visée de J.-B. Say, où seule importe l'achèvement de l'émancipation des hommes de l'emprise du politique.

Le secret de la croissance économique, de la prospérité et de la forme moderne de civilisation réside pour Verri dans la coexistence du besoin et de l'abondance. Le besoin concerne la marchandise que l'on recherche (demande) et l'abondance (offre) celle que l'on cède en échange: «A' mesure que les besoins augmentent la stimulation pour aug-

latin fort mal. Je puis dire, comme Jean Jacques Rousseau, que j'étais destiné à apprendre le latin toute ma vie et à ne le savoir jamais. Du reste les deux chefs de la maison étaient bons envers leurs élèves, ils soignaient les développements de leur corps et de leur esprit, et j'ai conservé un tendre souvenir des soins qu'ils m'ont donnés". Voir aussi Mss, Bibliothèque nationale, "carton $\mathrm{A}$ ", non paginé.

11 Toutes les citations ont été traduites par nous de l'original en italien.

12 Verri, "Dirò soltanto per quali mezzi l'economia politica ben diretta accrescerà la potenza di uno stato, (Verri, Meditazioni sulla economia politica, Scrittori Classici Italiani di Economia Politica, Parte Moderna, Milano, Custodi, 1804, vol. 15, p. 11). 
menter les marchandises aptes à être cédées en échange augmente également»r. ${ }^{13}$ Pour Verri une fois quitté l'état de sauvagerie au moment où naissent «de nouveaux besoins de nouvelles commodités» la nation «sera forcée d'augmenter proportionnellement son industrie et à multiplier la masse annuelle de ses produits». ${ }^{14}$ C'est le moteur de l'activité économique, plus largement cette croissance procède du développement de la communication entre les hommes sous toutes ses formes: «plus le transport devient facile, et alors plus la communication devient facile, plus les idées se multiplient, plus les besoins augmentent, plus le commerce augmente et plus l'agriculture d'un pays agricole augmente, car l'effet est toujours en raison de la cause; l'homme cultive en fonction de ses besoins, et il doit les satisfaire avec les produitsde son pays». ${ }^{15}$

Cette dynamique du commerce désigne ici toute l'activité de production y compris celle des services. Verri reprend l'analyse physiocratique pour laquelle le commerce regroupe toutes les étapes nécessaires à la transformation des richesses réelles en richesses pécuniaires. Une fois mise en marche la production prend un développement illimité. Cette dynamique repose sur le développement des besoins: «Et voilà comment à mesure que se multiplient les besoins d'une nation, naturellement le produit annuel du sol et de l'industrie nationale tend à augmenter». ${ }^{16}$ Ayant défini ce qui fonde la croissance économique, ici confondue avec le progrès général de la société, Verri aborde les lois qui commandent l'augmentation et la diminution de la richesse dans un Etat.

13 Verri, Meditazioni sulla economia politica, Scrittori Classici Italiani di Economia Politica, Parte Moderna, Milano, Custodi, 1804, vol. 15, "A misura che i bisogni crescono, cresce lo stimolo di aumentare le merci atte a cedersi in contraccambio”, p. 11.

14 Verri, Meditazioni sulla economia politica, Scrittori Classici Italiani di Economia Politica, Parte Moderna, Custodi, 1804, vol. 15, p. 12, "Allora sarà forzata ad accrescere proporzionatamente la sua industria e moltiplicare l'annua massa de' suoi prodotti".

15 Verri, Meditazioni sulla economia politica, Scrittori Classici Italiani di Economia Politica, Parte Moderna, Milano, Custodi, 1804, vol. 15, p. 19, "Quanto più si va rendendo facile il trasporto, tanto più si estende la comunicazione, tanto più si moltiplicano le idee, tanto più si accrescono i bisogni, tanto cresce il commercio e parallela cresce l'agricoltura in un paese agricol[o], essendo che l'effetto è sempre proporzionato alla cagione; l'uomo coltiva quanto domandano $i$ suoi bisogni $[. .$.$] ai quali deve corrispondere coi prodotti della sua terra".$

16 Verri, Meditazioni sulla economia politica, Scrittori Classici Italiani di Economia Politica, Parte Moderna, Milano, Custodi, 1804, vol. 15, p. 12, "Ed ecco come a misura che si moltiplicano $i$ bisogni di una nazione, naturalmente tendono ad accrescersi l'annuo prodotto del suolo e l'industria nazionale". 


\subsection{La critique des physiocrates}

$\mathrm{Au}$ chapitre III "Accrescimento e diminuzione della ricchezza di uno stato" ${ }^{17}$ il commence par se distinguer des physiocrates sur deux points essentiels, l'impôt et la prétendue classe stérile. Pour Verri la production doit être attribuée également à la classe des manufacturiers parce que «tous les phénomènes de l'univers qu'ils soient le résultat de l'action de la main de l'bomme ou des lois universelles de la physique, ne constituent pas une création actuelle mais une modification de la matière. Rapprocher et séparer sont les seuls éléments que l'esprit de l'bomme retrouve en analysant l'idée de la production». ${ }^{18}$

Il s'oppose à la définition donnée par Quesnay: «Il faut distinguer une addition de richesses réunies, d'avec une production de richesses, c'est à dire une augmentation par réunion de matières premières, et de dépenses en consommation, d'avec une génération ou création de richesses, qui forment un renouvellement et un accroissement réel de richesses renaissantes». ${ }^{19}$ En nette opposition à l'affirmation de Quesnay il écrit: «Tous les phénomènes de l'univers, qu'ils soient le résultat de la main de l'bomme, ou bien des lois universelles de la physique, ne constituent pas une création actuelle, mais uniquement une modification de la matière». ${ }^{20}$

Cette définition de toute activité de l'homme et de la nature, comme séparation et rapprochement suffit à elle seule, d'un point de vue logique, pour fonder le rôle fécond de la prétendue classe stérile des physiocrates. Il définit très exactement la valeur créé par la classe des manufacturiers: «la production de valeur est, donc, cette quantité du prix que contient le produit ou l'objet manufacturé, au-delà de la valeur de la

17 Verri, Meditazioni sulla economia politica, in Scrittori Classici Italiani di Economia Politica, Parte Moderna, Milano, Custodi, 1804, vol. 15, pp. 20-31.

18 Verri, Meditazioni sulla economia politica, Scrittori Classici Italiani di Economia Politica, Parte Moderna, Milano, Custodi, 1804, vol. 15, p. 21, "Tutti i fenomeni dell'universo, siano essi prodotti dalla mano dell'uomo, ovvero dalle universali leggi dellla fisica, non ci danno idea di attuale creazione, ma unicamente di una modificazione della materia. Accostare e separare sono gli unici elementi che l'ingegno umano ritrova analizzando l'idea della riproduzione".

19 Quesnay, Sur les travaux des artisans, novembre 1766, INED, T. 1, p. 690.

20 Verri, Meditazioni sulla economia politica, Scrittori Classici Italiani di Economia Politica, Parte Moderna, Milano, Custodi, 1804, vol. 15, p. 21. 
matière et des consommations faites pour la réaliser». ${ }^{21}$ Ce passage s'oppose à Quesnay pour qui le travail nécessaire à la production d'une marchandise est productif s'il dégage un surplus sur les frais de production. L'hypothèse de Quesnay pour les manufacturiers est que ceuxci ne dégagent pas de surplus ce qui suffit pour les caractériser comme classe stérile. $^{22}$

C'est après avoir défini le commerce considéré du point physique «comme le transport des marchandises d'un lieu dans un autre», dans un chapitre IV intitulé: «Principes moteurs du commerce et analyse $d u$ prix», ${ }^{23}$ que Verri définit ce que sont les éléments constitutifs du prix: "Quels sont donc les éléments qui forment le prix? Ce n'est certainement pas la seule utilité qui le constitue. Pour s'en convaincre il suffit de réfléchir que l'eau, l'air et la lumière du soleil n'ont pas de prix, et pourtant aucune autre chose n'est plus utile (...). De même la seule rareté d'une marchandise ne suffit pas pour lui donner un prix». ${ }^{24}$

Il ne suit pas une problématique de la valeur dissociée du prix. Il y a identité entre prix et valeur, le prix «est formé par deux principes réunis, besoin et rareté̀. ${ }^{25} \mathrm{Il}$ justifie le remplacement de la notion d'utilité par celle de besoin parce que: «dans la mesure où l'on parle de marché, c'est à dire de la permutation d'une chose avec une autre, par le mot

21 Verri, Meditazioni sulla economia politica, Scrittori Classici Italiani di Economia Politica, Parte Moderna, Milano, Custodi, 1804, vol. 15, p. 26, "La riproduzione di valore é, adunque, quella quantità di prezzo che ba la derrata o manifattura, oltre il valor primo della materia e la consumazione fattavi per formarla".

22 Pour la discussion de ce problème voir $\mathrm{Ch}$. Mercier, Thèse complémentaire, Lyon, 1977, "l'artisan détruit autant en subsistances, qu'il produit par son travail; il n'y a donc pas de multiplication de richesses dans la production des rouages de l'industrie, puisque la valeur de ces ouvrages n'augmente que du prix de la substance que ces ouvriers consomment", Quesnay, Grains, Janvier 1757, INED, T. 2, p. 496.

23 Verri, Metiazioni sulla economia politica, Scrittori Classici Italiani di Economia Politica, Parte Moderna, Milano, Custodi, 1804, vol. 15, pp. 32-52.

24 Verri, Meditazioni sulla economia politica, Scrittori Classici Italiani di Economia Politica, Parte Moderna, Milano, Custodi, 1804, vol. 15, pp. 35-36, "Quali sono dunque le elementi che formano il prezzo? Non é certamente la sola utilità che lo costituisca, per convincersi di questo basta riflettere che l'acqua, l'aria e la luce del sole non banno prezzo alcuno (...). Nemmeno la sola rarità di una merce basta a darle prezzo".

25 Verri, Metiazioni sulla economia politica, Scrittori Classici Italiani di Economia Politica, Parte Moderna, Milano, Custodi, 1804, vol. 15, p. 37, "Il prezzo delle cose vien formato da due principi riuniti, bisogno e rarità". 
de besoin, l'on ne veut pas donner un synonyme de dèsir, mais l'on entend uniquement la préférence que l'on donne à la marchandise que l'on recherche en comparaison de la marchandise que l'on veut céder». ${ }^{26}$

Le besoin signifiera «l'excès de l'estime que l'on fait de la marchandise que l'on dèsire, en comparaison de celle que l'on veut céder $\gg .{ }^{27}$ Lorsqu'il y a transaction l'estime que l'on fait est identique de part et d'autre, le besoin qui entre dans la formation du prix du produit est un vrai désir. L'évaluation de ce besoin n'est pas l'intensité du désir particulier de celui qui recherche telle marchandise particulière mais la plus ou moins grande recherche de la plus grande partie des participants à l'échange, ce qui va se mesurer par l'utilité commune. De là il découle que «le prix sera en raison du besoin commun et de la rareté». ${ }^{28}$

\section{L'ENJEU DE LA DISCUSSION SUR LA VALEUR ${ }^{29}$}

\subsection{Valeur et vision d'ensemble}

La plupart des ouvrages en français qui traitent de J.-B. Say font remonter l'origine de sa théorie de la valeur à l'influence de Condillac (1715-1780) ou bien encore à Turgot.(1766). Il y a de bonnes raisons de penser que pour ces filiations la véritable origine

26 Verri, Meditazioni sulla economia politica, Scrittori Classici Italiani di Economia Politica, Parte Moderna, Milano, Custodi, 1804, vol. 15, p. 37, "Riflettasi che quando si parla di mercato, ossia di permutazione di una cosa coll'altra col nome di bisogno, non s'intende già un sinonimo del desiderio, ma s'intende unicamente "la preferenza che si dà alla merce che si ricerca, in paragone della merce che si vuol cedere". Dunque bisogno significherà "l'eccesso della stima che si fa della merce che si desidera, in paragone di quella che si vuol cedere".

27 Verri, Meditazioni sulla economia politica, Scrittori Classici Italiani di Economia Politica, Parte Moderna, Milano, Custodi, 1804, vol. 15, p. 38: dunque bisogno significherà l'eccesso della stima che si fa della merce che si desidera, in paragone di quella che si vuol cedere.

28 Verri, Meditazioni sulla economia politica, Scrittori Classici Italiani di Economia Politica, Parte Moderna, Milano, Custodi, 1804, vol. 15, p. 38.

29 Sur les théories de la valeur chez les classiques voir Claude Mouchot, Economie politique, Paris, Economica, pp. 289-325, A. Lapidus, Le détour de valeur, Paris, Economica, 1986, pp. 15-70. 
de sa position en matière de théorie de la valeur se trouve chez l'auteur qu'il cite au tout début de la première édition du Traité et qu'il continuera de citer tout au long des rééditions et des ajouts: le comte Pietro Verri. Ce qui ne contredit en rien le fait que Say assume un «triple héritage: (celui) des mercantilistes, (...) des physiocrates (...); enfin d'Adam Smith». ${ }^{30}$

Say, cherche à prouver, que la production et l'ensemble de l'activité économique est une immense permutation de valeurs, à travers une immense circulation de produits. Cette analyse est soutenue par un objectif plus vaste qui prolonge toute son action au sein du groupe des idéologues dont il est l'un des acteurs important; «il fallait, enfin», écrit-il, «rendre la doctrine tellement populaire, que tout bomme doué d'un sens droit pût la saisir dans son ensemble et dans ses détails et en appliquer les principes à toutes les circonstances de la vie». ${ }^{31} \mathrm{Il}$ considère la production et l'échange comme un seul et même processus unitaire qui lui impose de trouver une définition de la valeur qui élimine toutes les difficultés logiques.

Pour lui la société procède du consentement des individus libres, et en cela il est fidèle à son idéal républicain. Il ne peut y avoir de règles procédant d'un ordre supérieur mais seulement la libre connaissance acquise par l'expérience et l'observation. Son ambition est de donner une forme rationnelle à l'ordre républicain et que cet ordre repose sur cette classe industrieuse qui doit tout à ses talents et à son labeur. Il s'agit pour Say d'assumer un héritage et de résoudre en termes économiques les principaux problèmes politiques et sociaux de l'époque. ${ }^{32}$

On peut s'interroger à ce propos pour savoir si Say est motivé par son échec politique dans ses relations avec le premier consul Bonaparte, dont il espérait beaucoup, ou bien si sa vision plus fondamentale consistait de toute façon à concevoir un monde dans lequel le rôle de l'Etat soit le plus réduit possible. Les deux interprétations ne sont pas exclusives l'une de l'autre. Cependant nous pouvons avancer ici le fait

30 Say, TEP, Ge édit., Préface de G. Tapinos, p. VI.

31 Say, TEP, 6e édit., Discours Préliminaire, pp. 34-35, Paris, Calmann-Lévy, 1972.

32 Comme l'indique à propos, cette fois des physiocrates, J. Cartelier, introduction à Quesnay, Physiocratie, Paris, Garnier, Flammarion, 1991, p. 11. 
que ses déboires avec Bonaparte et les régimes politiques avant et après ont très probablement du renforcer chez lui la volonté d'émanciper l'économie politique de l'emprise de L'Etat pour émanciper les hommes.

\subsection{Valeur et commerce}

L'enjeu de la définition du commerce comme transport doit être précisé. Ce passage apparaît souvent au lecteur comme d'une grande banalité sauf si l'on s'intéresse à l'auteur que Say cite à l'appui de sa démonstration: Pietro Verri. La notion de transport doit être entendue ici dans le sens large de rapprocher de façon générale les acheteurs des vendeurs. Elle permet à Say, comme chez Verri, de donner à toute activité commerciale, ou liée au processus de la distribution et de la commercialisation des marchandises, un rôle productif. La distinction Smithienne entre travail productif et travail improductif est éliminée.

Pour fonder et prouver le caractère erroné de cette distinction il fallait aussi disposer d'une définition de la valeur qui permette de fonder logiquement la cohérence des rapports entre toutes les activités économiques et la répartition, des revenus entre les différents acteurs. La définition du fondement de la valeur comme valeur utilité va lui permettre de lier en un tout cohérent toutes les parties de l'activité économique. Pour établir cette "vérité" il commence d'abord par dire que la production des richesses n'est pas «une création de matière mais une création d'utilité». Toutes les difficultés quand à la répartition des revenus disparaissent avec l'abolition de la distinction du productif et de l'improductif, de ce qui est matériel ou immatériel. Enfin Say se satisfait d'une définition de la valeur à partir de la seule utilité en abandonnant toute explicitation du rôle de la rareté.

On peut en trouver la raison dans plusieurs éléments. Tout d'abord il s'oppose fermement à l'utilisation systématique de «l'esprit de géométrie» ${ }^{33}$ et à l'utilisation des mathématiques. Toute formulation qui lui semble trop. rigide et trop. formelle est écartée de même que toute problématique reliée à la notion d'équilibre. Sa conviction pro-

33 Voir Y. Breton, Les économistes français et les questions de méthode, pp. 389420 in L'économie politique en France au XIXe siècle, sous la direction de Y. Breton, M. Luftalla, p. 390, Paris, Economica, 1991. 
fonde sur le plan méthodologique est que la connaissance scientifique ne peut progresser en économie qu'à partir de l'observation et de l'expérience. ${ }^{34}$ Rappelons ce que sont les éléments de la définition de la valeur utilité par Say. ${ }^{35}$ Pour lui un bien économique est un bien qui a une valeur sociale, qui est une richesse sociale, il est produit et a un coût. La valeur échangeable des choses est la valeur utilité, mesurée par le prix exprimé en quantité de monnaie ou bien en toute autre quantité de marchandise donnée en échange.

L'objectif de Say est d'appréhender dans toute son étendue le phénomène de la production, le processus de formation des richesses sociales. C'est la compréhension de ce processus qui conditionne la possibilité pour les hommes d'accéder à une vie décente. Dans cette perspective l'analyse conduite par Smith, qui constitue le point de départ de Say, conserve des éléments de la conception physiocratique qui lui interdisent d'inclure la totalité des activités économiques. En particulier le rôle des différents facteurs de production (terre,travail, capital) Smith faisant du travail et de la division du travail la source principale de la richesse.

L'examen des notes de Say, portées sur son exemplaire personnel de la Richesse des Nations de Adam Smith, permet d'avancer dans l'analyse du processus de formation de sa pensée. ${ }^{36}$ Les notes qui couvrent l'exemplaire de la Richesse des Nations sont de deux ordres: celles qui critiquent différents passages et celles qui résument. Ces notes ont été portées à partir de l'acquisition de l'exemplaire en $1789^{37}$ et très probablement fortement développées durant la rédaction du Traité entre 1799 et 1803, période au cours de laquelle Say est membre de la section des finances du Tribunat.

Elles permettent de saisir ce qu'a été en partie la formation de sa

34 Voir P. Steiner, "L'économie politique pratique contre les systèmes: quelques remarques sur la méthode de J.-B. Say”, Revue d'Economie Politique, 1990, $100^{\circ}$ année, $\mathrm{n}^{\circ}$ 5, pp. 664-687. et A. Bonnafous, Le siècle des ténèbres de l'économie, Economica, 1989, pp. 69-70.

35 On trouvera une présentation de ces propositions in M. Luftalla, JeanBaptiste Say 1767-1832. Le fondateur, in L'économie politique en France au XIXe siècle, sous la direction de Y. Breton et M. Luftalla, Paris, Economica, 1991, pp. 13-31.

36 Ces notes ont été éditées en 1980 par Hitoshi Hashimoto in KSU Economic and business review, vol 7, fascicule 5, p. 53-81 et vol 9, p. 31-133 GrandeBretagne, 1980 sous le titre: Notes inedites qui couvrent les marges de la richesse des nations et qui la critiquent: redigées avec une introduction de Hitoshi Hashimoto. 
pensée sur la théorie de la valeur. La première note critique figure sur la première page de l'introduction de l'ouvrage de Smith: «Le travail est le seul fondement de la valeur des choses» (je crois que c'est une erreur). ${ }^{38}$ Ici la première partie résume le point de vue de Smith, ce qui est entre parenthèses exprime le point de vue de Say. Dans la mesure où il s'agit de notes on peut faire l'hypothèse qu'il s'agit là de l'expression de l'intuition de Say indépendamment de toute lecture ou influence, ${ }^{39}$ le caractère hésitant de l'affirmation plaide en ce sens. Les notes qui suivent confirment le premier doute de Say: «Smith me paraît commettre en ce chapitre une double erreur. $1^{\circ}$ Tous les biens du monde n'ont pas été achetés par le travail de l'bomme. La nature a une part dans certains produits et son travail donne une valeur additionnelle à celui de l'bomme». ${ }^{40}$

La critique se pousuit à propos du capital et de la distinction entre productif et improductif il écrit: «Mais la thèse que Smith soutient ici rentre dans son système qui range dans la classe des travaux improductifs, ceux qui ne sont productifs que d'une utilité ou d'une jouissance incorporelle, comme le travail d'un magistrat, d'un musicien. Ce système tient un peu de celui des économistes; et ceux-ci pouvaient s'en servir pour combattre Smith lorsqu'il prétend que le travail des manufactures est productif. En effet que produit-il? pas un atome; mais seulement une utilité, une valeur de plus dans la chose travaillée, et par conséquent une quantité purement immatérielle». ${ }^{41} \mathrm{La}$ suite des notes comporte une critique de la place trop. réduite que Smith accorde au commerce: «Cependant Tyr, Carthage, Venise se sont enrichies principalement par le commerce extérieur: Smith méconnaît trop. la faculté productive de l'industrie commerçante» ${ }^{42}$ et plus loin il précise «Smith oublie le principal produit du com-

37 Voir sur ce point l'intoduction de Hitoshi Hashimoto in KSU Economic and business review, vol 7, fascicule 5, p. 53-56 Grande-Bretagne,1980 à: Notes inedites qui couvrent les marges de la richesse des nations et qui la critiquent.

38 Hitoshi Hashimoto, op.cit., p. 67.

39 Les manuscrits de Say ne comportent en règle générale aucune indication de date, et pour les notes qui figurent sur son exemplaire personnel conservé à l'Institut de France aucune indication ne permet de se prononcer avec certitude sur la date de leur rédaction. Mais la date de son acquisition (1789) plaide dans le sens de l'antériorité sur d'autres lectures.

40 Hitoshi Hashimoto, op.cit., p. 68.

41 Hitoshi Hashimoto, op.cit., p. 74.

42 Hitoshi Hashimoto, op.cit., p. 77. 
merce: c'est à dire l'augmentation de valeur donnée à la marchandise indigène en l'exportant et à la marchandise étrangère en l'important»r. ${ }^{43}$

Abordant la place de Smith dans les Préliminaires du Traité. Say énonce une série de critiques, nous ne retiendrons ici que celles qui intéressent directement notre propos sans vouloir être exhaustif à cet égard. Il commence tout d'abord par dire qu'il démontra que la richesse était la valeur échangeable des choses; qu'on était d'autant plus riche qu'on avait plus de choses qui eussent de la valeur. ${ }^{44}$ Ayant crédité Smith d'avoir posé le fondement d'une appréciation correcte de la définition de la richesse, il lui reproche ensuite: «d'avoir en cherchant ce qui donne cette valeur trouvé que c'est le travail de l'bomme qu'il aurait dî appeler industrie, parce que le mot industrie embrasse des parties que le mot travail n'embrasse pas». ${ }^{45}$ Ensuite il aborde la caractérisation des produits et leur capacité à posséder de la valeur et donc à fonder une définition productive de tous les actes économiques. Il écrit «Smith a borné le domaine de cette science en réservant le nom de richesses aux valeurs fixées dans des substances matérielles». ${ }^{46}$ Say vise la distinction de Smith entre productif et improductif et en particulier le rôle de la classe commerçante. Pour lui celui-ci (...) «ne donne que des idées confuses sur la manière dont le commerce est productif; ce qui l'empêche de déterminer avec précision pour quelle raison et jusqu'à quel point la facilité des communications contribue à la production» ${ }^{47}$

Notons ici que plus tard Say créditera les physiocrates et non Smith d'avoir donné uhne définition correcte de la monnaie: «Je conviens que c'est aux économistes français du $18^{\circ}$ siècle que nous avons l'obligation d'avoir formé Adam Smith. Ce sont eux qui ont nettement professé et prouvé que la richessse ne consiste pas dans l'agent de la circulation (circulating médium) mais dans les choses que l'on échange», ${ }^{48}$ on peut pour occasion à propos de la valeur être surpris de ce que Say n'ait pas repris la proposition de Tooke de définir la valeur par l'utilité et la rareté, voici l'extrait de la lettre de Tooke: «Dans quelques unes de

43 Hitoshi Hashimoto, op.cit., p. 79.

44 Say, TEP, 6e, p. 26, Paris, Calmann-Lévy, 1972.

45 Say, TEP, 6e, p. 27, Paris, Calmann-Lévy, 1972.

46 Say, TEP, 6e, pp. 30-31, Paris, Calmann-Lévy, 1972.

47 Say, TEP, 6e, p. 31, Paris, Calmann-Lévy, 1972.

48 Mss, BN, fonds Say. 
nos dernières réunions de la Société d'Economie Politique, la définition suivante des richesses a reçu l'assentiment d'une portion considérable de ses membres, et l'on est convenu de l'adopter comme exprimant la signification la plus générale de ce mot: Objet calculés pour l'usage ou la jouissance, et existant en quantité limitée. (..) Les conditions ainsi réclamées, savoir, l'utilité d'un part et d'autre part la quantité limitée, me paraissent simplifier la considération du sujet qu'on a jusqu'ici mal à propos compliqué»». ${ }^{49}$ Face à cet argument Say ne dit rien et repousse la propositon dans sa lettre qui suit. ${ }^{50}$

\section{SAY ET VERRI: EMPRUNTS ET RUPTURES}

\subsection{Valeur-utilité et commerce}

J.-B. Say reprend pour définir la valeur la seule base de l'utilité sans faire de référence à la rareté, il s'appuit le plus souvent sur la loi de l'offre et de la demande. Si l'on reprend la définition qu'il donne dans l'épitômé de la cinquième édition du Traité (1826) on retrouve une définition proche de celle de Verri du prix comme prix de marché et l'absence de distinction entre prix naturel et prix de marché: «Le prix courant est celui auquel, en, chaque lieu, une chose trouve des acquéreurs» ${ }^{51}$ Lorsqu'il introduit la notion de prix originaire il traite du prix du produit en terme de coût de production c'est-à-dire la limite en dessous de laquelle il ne saurait être vendu en permanence ou pour une longue durée.

Considèrant que les activité du commerçant et du savant sont productives, il doit rejeter la conception de la valeur qu'il trouve chez Smith et la distinction que celui-ci opère entre valeur d'usage et valeur d'échange. Pour lui il n'y pas de monde réel caché par un voile monétaire, valeur et prix peuvent coincider. ${ }^{52}$ Say part de Verri pour qui:

49 Lettre de Tooke à J.-B. Say, du 8 mars 1828, in Oeuvres Diverses, Guillaumin, 1846, pp. 531-532.

50 Lettre de Tooke à J.-B. Say, du 8 mars 1828, in Oeuvres Diverses, Guillaumin, 1846, pp. 531-532. pp. 533-539.

51 Say, TEP, $5^{\circ}$, tome III, p. 306.

52 Sur ce point les analyses de Say ont variées, dans la première édition du Traité (1803) il semble reprendre l'opposition entre prix naturels et prix courants et la 
l'«échange n'était qu'un échange de valeur». Il trouve chez ce dernier une définition du commerce comme transport des marchandises.

Le premier point lui permet de donner un fondement à la caractérisation des activités de commerce comme véritablement utiles et de les inclure dans la production: «Le courtier rapproche les vendeurs des acheteurs. Le banquier, le changiste, fournissent des letters - de - change, payables dans d'autres lieux que ceux où l'on est, ou, des monnaies étrangères nécessaires pour payer le prix des achats (...) Tous font le commerce» ${ }^{53} \mathrm{Ce}$ pas supplémentaire est indispensable à ses yeux pour achever le travail initié par Smith: rompre complètement avec les erreurs de la physiocratie. Le métier des manieurs d'argent est de l'ordre du commerce, la monnaie est une marchandise comme les autres.

Toutefois il se sépare de P. Verri qui fait de la monnaie une «marchandise universelle» sans laquelle on ne peut pas avoir idée de la valeur, elle assure la cohérence de tous les actes économiques et permet aux activités humaines de se compenser et se mesurer. Il est significatif que Verri commence les "Méditations" par trois chapitres ${ }^{54}$ qui traitent des sociétés sans monnaie puis des sociétés avec commerce et monnaie, avant d'en venir au commerce proprement dit et à la dynamique économique.

\subsection{Utilité et création de richesse}

Pour Say l'activité de production est déterminante, elle doit d'abord être prise en compte pour éclairer tout le reste. Ainsi il situe la production non pas comme une pure création de matière car: «On ne crée pas des objets: la masse des matières dont se compose le monde, ne saurait augmenter ni diminuer. Tout ce que nous pouvons faire, c'est de reproduire ces matières sous une autre forme qui les rende plus propres à un usage quelconque qu'elles n'avaient pas, ou seulement qui augmente

thèse de la gravitation. Cette lecture disparaît dans l'Epitômé de la $5^{\circ}$ édition après que ses notes à la traduction par Constancio des principes de Ricardo aient marqué cet abandon, voir les notes de J.-B. Say republiées dans la nouvelle traduction des Principes de l'économie politique et de l'impôt, édition anglaise de 1821, présentation de F.-R. Mahieu, Paris, Flammarion, 1993, pp. 455-457.

53 Say, TEP, 1ère, tome 1, p. 148.

54 Verri, Meditazioni sulla economia politica, Scrittori Classici Italiani, di Economia Politica, Parte Moderna, Milano, Custodi, 1804, vol. 15, pp. 9-31. 
l'utilité qu'elles pouvaient avoir. Alors il y a création non pas de matière, mais d'utilité, et comme cette utilité leur donne de la valeur, il y a production de richesses». ${ }^{55}$ Ce que Verri avait dit de façon plus explicite comme nous l'avons vu plus haut.

Verri a développé une analyse en opposition avec celle des physiocrates où il défend très vigoureusement le rôle productif de l'industrie, des manufactures, à côté de l'agriculture. Sur ce terrain là ils ne pouvaient que s'accorder Verri écrit: «Des cités entières et des Etats entiers ne reposent pas sur autre chose que sur la production de cette très féconde "classe stérile", dont la reproduction comprend la valeur de la matière première, la consommation proportionnelle des mains utilisées, et en outre cette portion qui enrichit celui qui a créé l'entreprise et qui s'y emploie avec grands talents..$^{56}$

Verri caractérise les opérations de production comme modification de la forme des matières déjà existantes et non pas comme création de matière: «rapprocher et séparer sont les seuls éléments que l'esprit bumain retrouve en analysant l'idée de reproduction». ${ }^{57}$ Say reprend ce point en le développant dans le chapitre II «Des différentes sortes d'industrie et comment elles concourent à la production». Pour lui l'industrie agricole se borne à recueillir les fruits de la nature, l'industrie sépare, mélange, façonne les produits de la nature et quand à l'industrie commerciale elle met les objets à notre portée. ${ }^{58} \mathrm{Il}$ place l'accent sur la question de la production «C'est ainsi qu'il faut entendre le mot production en économie politique, et dans tout le cours de cet ouvrage. La production n'est point une création de matière, mais une création d'utilité».".

Il y a une autre différence qui doit être notée: pour Verri l'économie politique est l'affaire du conseiller du Prince et ses Méditations

55 Say, TEP, tome 1, 5e édition, pp. 6-7.

56 P. Verri, Meditazioni sulla economia politica, Scrittori Classici Italiani, di Economia Politica, Parte Moderna, Milano, Custodi, 1804, vol. 15, p. 22, "Delle intiere città e degli stati intieri campano non d'altro che sul prodotto di questa fecondissima classe sterile, la di cui riproduzione comprende il valore della materia prima, la consumazione proporzionata delle mani impiegatevi, e di più quella proporzione che fa arricchire chi ha intrapresa la fabbrica e chi vi si impiega con felice talento". Précisons à ce propos que K. Marx cite Verri contre les physiocrates sur ces mêmes passages, Théories sur la plus-value, T. I, pp. 61-62, Paris, Editions sociales, 1974.

57 Verri, Meditazioni sulla economia politica, Scrittori Classici Italiani, Custodi, 1804, T 15,p. 21, "Accostare e separare sono gli unici elementi che l'ingegno umano ritrova analizzando l'idea della riproduzione". 
contiennent deux chapitres qui concluent son oeuvre majeure sur ce que doit être un ministre de l'économie et ce que doit être un ministre des finances.$^{60}$ Say s'adresse à la la classe mitoyenne, celle des cadres de l'industrie, du commerce, de l'administration. Cependant ils partagent l'un et l'autre cette maxime des Lumières: «L'erreur seule, les opinions enchâेnent les hommes et conduisent toutes les nations à la misérable stérilité». ${ }^{61}$

\subsection{La monnaie}

Dans le chapître 2 de ses "Meditazioni sull'economia politica" Pietro Verri définit la place de la monnaie par rapport au commerce: «Afin que s'établisse un échange commercial stable et réciproque entre homme et homme, et encore plus entre Etat et Etat, il était donc nécessaire que l'on trouve d'abord le moyen d'avoir une idée universelle de la valeur, et que l'on trouve une marchandise incorruptible, divisible, acceptée par tous et toujours, facile à conserver, et à transporter, apte en somme à être échangée contre n'importe quelle autre marchandise. Avant l'invention de la monnaie il n'était donc pas physiquement possible qu'un échange stable et réciproque existe entre les hommes, et entre les peuples. Parmi les nombreuses définitions, qu'il m'est arrivé de lire, portant sur la monnaie, je n'en ai trouvé aucune qui me paraisse correspondre exactement aux caractéristiques de la monnaie»». ${ }^{62}$ Ici Verri en dehors des carac-

58 Say, TEP, 5e édition, tome I, p. 10.

59 Say, TEP 5e édition, tome I, p. 7.

60 Verri, Meditazioni sulla economia politica, Scrittori Classici Italiani di Economia Politica, Parte Moderna, Milano, Custodi, 1804, vol. 15, pp. 330-337.

61 Verri, Meditazioni sulla economia politica, Scrittori Classici Italiani di Economia Politica, Parte Moderna, Milano, Custodi, 1804, vol. 15, p. 337, "L'errore solo, le opinioni incatenano gli uomini e guidano le intiere nazioni alla squallida sterilità".

62 Verri, Meditazioni sulla economia politica, Scrittori Classici Italiani di Economia Politica, Parte Moderna, Milano, Custodi, 1804, vol. 15, pp. 13-14, Del danaro, e come accresca il commercio: "Accioché s'introducesse una stabile e reciproca comunicazione di commercio fra uomo e uomo, e molto più fra stato e stato era necessario adunque che primieramente si ritrovasse il mezzo per avere una idea universale del valore, e si ritrovasse una merce incorrutibile, divisibile, accettata sempre da ognuno, facile a custodirsi e a trasportarsi, atta in somma a potersi cedere in contraccambio di ogni altra merce. Prima dell'invenzione del danaro non era perciò fisicamente fattibile che s'introducesse una reciproca e stabile comunicazione fra nomo e uomo, fra popolo e popolo. Fra le molte definizioni, che mi è accadutto di leggere, date al danaro, non ne ho trovata alcuna la quale mi sembri corrispondere esattamente all'indole di esso". 
téristiques classiques de la monnaie, de divisibilité, inaltérabilité, grande valeur sous un faible volume met l'accent sur le fait que l'existence de la monnaie permet d'avoir une idée générale de la valeur et surtout d'établir un échange régulier.

Les caractéristiques que Say énonce à propos de la marchandisemonnaie se retrouvent en partie quoique de façon plus concise chez Verri. Pour ce dernier la monnaie est «la marchandise universelle: c'est à dire cette marchandise qui du fait de son acceptation universelle», ${ }^{63} \mathrm{de}$ son faible volume qui en rend le transport facile, du fait de sa divisibilité et de son inaltérabilité «est universellement reçue en échange de n'importe quelle marchandise particulière». ${ }^{64}$ Verri en considérant la monnaie sous cet aspect la définit de façon telle que «l'on en a une idée qui n'appartient qu'à elle, et qui en montre exactement tous les usages». Il en donne ensuite la définition logique par genre et différence ${ }^{65}$ comme le voulait les scolastiques: «le genre est marchandise, le spécifique est universel. Les contrats d'achats et de vente deviennent de simples constats de permutation et sont facilement compris». ${ }^{66}$

On retrouve l'approche de J.-B. Say quand Verri insiste sur l'échangeabilité générale que doit posséder la marchandise choisie comme monnaie. Verri parle de marchandise universelle pour désigner la monnaie, le terme même sera repris en opposition plusieurs fois par

63 Verri, Meditazioni sulla economia politica, Scrittori Classici Italiani di Economia Politica, Parte Moderna, Milano, Custodi, 1804, vol. 15, p. 16.

64 Verri, Meditazioni sulla economia politica, Scrittori Classici Italiani di Economia Politica, Parte Moderna, Milano, Custodi, 1804, vol. 15, pp. 16-17.

65 Le genre désigne en logique l'Idée générale ou classe englobant plusieurs espèces, la différence spécifique est le caractère par lequel une espèce se distingue des autres espèces du même genre. P. Foulquié, avec la collaboration de R. Saint-Jean, Dicionnaire de la langue philosophique, Paris, PUF, 1986, p. 176-177 et 300-301.

66 Verri, Meditazioni sulla economia politica, Scrittori Classici Italiani di Economia Politica, Parte Moderna, Milano, Custodi, 1804, vol. 15, pp. 16-17: "il danaro è la merce universale, cioè a dire, è quella merce la quale per la universale sua accettazione, per il poco volume che ne rende facile il trasporto, per la comoda divisibilità, e per la incorrutibilità sua è universalmente ricevuta in iscambio di ogni merce. Mi pare che riguardando il danaro sotto di questo aspetto venga definito in modo che se ne ba un'idea propria a lui solo, che esattamente ce ne dimostra tutti gli uffici. Questa mi pare la definizione logica per genere e differenza, quale scolasticamente si vuole: l'attributo generico é merce, lo specifico universale I contratti di compra e vendita ritornano al semplice stato di permutazione ed a più facile intelligenza". 
J.-B. Say. Cependant on peut souligner qu'il place l'accent très fortement sur l'aspect consensuel du choix de la marchandise monnaie, échappant ainsi à l'emprise du politique. Les marchandises s'échangent contre des marchandises, la monnaie ne représente pas la totalité de la richesse sociale. Une fois éliminée la fausse identification entre richesse et monnaie, l'analyse est simplifiée, la production se ramène à un échange généralisé. Le fil conducteur de l'échange et du statut de la monnaie nous paraît être sur la base de l'analyse des textes tout autant chez Verri que chez Turgot. ${ }^{67}$

Ce n'est pas chez Turgot que Say emprunte une bonne partie de sa vision générale du rôle de la monnaie comme agent de permutation des différentes marchandises. On doit considérer par ailleurs qu'une bonne partie de l'analyse de Turgot et de Verri, qui écrivent à la même époque et qui sont confrontés aux mêmes types de problèmes, sont issues des scolastiques ${ }^{68}$ qui ont réalisé le travail préalable d'analyse sans lequel l'émancipation de l'économique du politique deviendrait très difficilement pensable.

L'introduction de la monnaie se traduit par une modification dans la langue: «Avant l'invention de la monnaie on ne pouvait pas avoir l'idée de vendeur et d'acheteur, mais seulement d'offreur et d'adhérant à l'échange. Après l'introduction de la monnaie celui qui cherche à échanger la marchandise universelle contre une autre marchandise a été appelé acheteur, et celui qui cherche à échanger une chose quelconque contre la marchandise universelle s'appelle le vendeur» ${ }^{69}$ Ce que Say reprend à sa

67 Il est cependant vrai que l'on peut plaider avec beaucoup. de bons arguments une continuité pus forte entre Say et Turgot sur la question de la monnaie.Cette continuité J.-B. Say lui même la revendique il écrit: "Turgot développa la théorie de la monnaie et cette vérité neuve alors que la monnaie ne remplis pas son office en vertu de l'autorité du gouvernement, qu'elle n'est pas plus un signe représentatif que la marchandise qu'elle achète; il distingua fort bien la somme des monnaies de celle des capitaux et ses principes sur ce qu'on appelle mal à propos l'intérêt del'argent sont excellents" Cours Complet, $1^{\circ}$ édition, pp. 384-385.

68 A. Lapidus, Le détour de valeur, Paris, Economica, pp. 17-54.

69 Verri, Meditazioni sulla economia politica, Scrittori Classici Italiani di Economia Politica, Parte Moderna, Milano, Custodi, 1804, vol. 15, p. 33: "Prima dell'invenzione del danaro non potevano aversi le idee di compratore e di venditore, ma soltanto di proponente, e di aderente al cambio. Dopo l'introduzione del danaro ebbe il nome di compratore colui che cerca di cambiare la merce universale con un'altra merce, e colui che cerca di cambiare una cosa qualunque colla merce universale si chiamò venditore". 
manière en écrivant: «L'échange d'un produit quelconque contre la marchandise répétant plus souvent que toute autre, on et lui a donné un nom particulier. Recevoir de la monnaie en échange, c'est vendre; en donner, c'est acheter». ${ }^{70}$

Pour Say comme pour Verri, la marchandise-monnaie n'est pas mesure des valeurs, pour Verri pour faire exactement le calcul de valeur entre deux sociétés sans communication du fait de leur éloignement géographique, ou de leur éloignement dans le temps, «il conviendrait de disposer d'une troisième quantité inaltérable à laquelle on puisse les comparer comme l'extension inaltérable du bras et la gravité constante des anches transportées et comparées donneront le moyen pour calculer les vrais rapports entre deux hauteurs ou deux poids éloignés; mais cette quantité inaltérable pour comparer les valeurs n'existe pas et il est impossible qu'elle existe; parce que la monnaie elle-même bien qu'elle soit marchandise universelle, est tantôt de valeur plus haute tantôt plus basse, et par conséquent incapable de servir de mesure». ${ }^{71}$

Dans l'analyse de la monnaie, Verri considère celle-ci comme une marchandise identique aux autres sans caractéristiques spéciales. Il refuse d'y voir la représentation de la valeur ou le moyen de paiement exclusif: «Certains voient dans la monnaie la représentation de la valeur des choses, mais la monnaie n'est pas une chose, c'est un métal, dont la valeur est également représentée par ce que l'on donne en échange de celui-ci; et cette propriété de représenter la valeur est commune à toutes les autres marchandises qui font l'objet d'un échange. D'autres considèrent la monnaie comme un gage (créance), et un moyen pour obtenir les marchandises, mais sous cet aspect également les marchandises sont ellesmêmes un gage et un moyen pour obtenir d'autres marchandises. D'autres définissent la monnaie comme la commune mesure des choses et ainsi ils

70 Say, TEP, 1ère édition, tome 1, p. 420.

71 Verri, Meditazioni sulla economia politica, Scrittori Classici Italiani di Economia Politica, Parte Moderna, Milano, Custodi, 1804, vol. 15, p. 147: "Per fare esattamente il calcolo del valore fra due società incomunicanti per distanza di luogo, o di tempo, converrebbe avere una terza quantità inalterabile a cui paragonarli come l'inalterabile estensione del braccio e la gravità costante delle ancia trasportate e paragonate daranno il mezzo per calcolare i veri rapporti fra due altezze o due pesi distanti; ma questa quantità inalterabile per paragonare i valori non vi é, ne é possibile che vi sia; perché il danaro istesso, sebbene sia merce universale, é ora di maggiore ed ora di valor minore, e percio incapace di servire di misura". 
oublient que la monnaie a une valeur et qu'elle est matière première de beaucoup. de produits manufacturés». ${ }^{72}$

J.-B. Say reprend la même argumentation: «En premier lieu ce n'est pas la quantité d'argent qui peut être la mesure d'une valeur, c'est sa valeur. Il y a de l'analogie, de la ressemblance entre la valeur d'une chose et celle d'une autre; mais il n'y en a aucune entre le poids ou la longueur d'une chose et la valeur d'une autre». ${ }^{73}$

La relation entre prix et monnaie est posée chez Say comme chez Verri: «Parmi les choses qui peuvent être données en échange de celles qu'on veut acquérir, se trouve la monnaie. La quantité de monnaie que l'on consent à donner pour obtenir une chose, se nomme son prix». ${ }^{74}$ Verri écrit: «Le prix, précisément parlant, signifie la quantité d'une chose que l'on donne pour en avoir une autre (...) Chez nous qui utilisons la marchandise universelle, le mot prix signifie la quantité de marchandise universelle que l'on donne pour azvoir une autre marchandise». ${ }^{75}$

72 Verri, Meditazioni sulla economia politica, Scrittori Classici Italiani, di Economia Politica, Parte Moderna, Milano, Custodi, 1804, vol. 15, pp. 14-17: "Alcuni ravvisano nel denaro la rappresentazione del valor delle cose: ma il danaro non é cosa, é un metallo, di cui il valore é egualmente rappresentato da quanto si dà in contraccambio di esso; e questa proprietà di rappresentare il valore é comune a tutte le altre merci generalmente contrattate. Altri ravvisano il danaro come un pegno, e mezzo per ottenere le merci, ma sotto di questo aspetto egualmente pure le merci sono un pegno e mezzo per ottenere il danaro e ogni merce é pegno e mezzo per ottenere un'altra merce Altri definiscono il danaro la comune misura delle cose, e con ciò dimenticano che il danaro ba un valore ed é materia prima di molte manifatture, e qualunque cosa che abbia il valore misura parimente ed é misurata da ogni altra cosa di valore. [...] Queste definizioni dunque non competono privatamente al danaro, o non ne comprendono tutte le qualità. L'errore si é comunemente adottato perché si é voluto considerare il danaro per qualche cosa di piu che semplice metallo. Il danaro ba un impronto, ma non riceve valore dall'impronto. Il danaro é la merce universale cioé a dire, é quella merce la quale per la universale sua accettazione, per il poco volume che ne rende facile il trasporto, per la comoda divisibilità e per la incorrutibilità sua é universalmente ricevuta in iscambio di ogni merce particolare".

73 Say, TEP, 1ère édition, tome 1, p. 472. Il écrit plus loin, tome 1, p; 478, "Le métal précieux, qu'il soit monnayé ou non, n'est donc qu'une marchandise dont la valeur est arbitraire et se règle à chaque marché qu'on fait, par un accord entre le vendeur et l'acheteur; il ne peut par conséquent remplir l'office d'une mesure dont le premier caractère est d'être invariable".

74 Say, TEP, 5ème édition, tome 1, p. 4.

75 Verri, Meditazioni sulla economia politica in Scrittori Classici Italiani di 


\section{Jean-Baptiste Say, Pietro Verri et La Loi des débOuchés}

La controverse sur la loi des débouchés est célèbre. Elle a donné lieu à de multiples articles et à un renouveau autour des années cinquante. Avant de s'interroger sur la paternité de cette loi il est nécessaire d'établir de façon aussi détaillée que possible la position de JeanBaptiste Say en 1803. W.J. Baumol ${ }^{76}$ a relevé que le contenu même de la loi ne figure pas dans le chapitre intitulé de «Des débouchés», que plus qu'un énoncé c'est surtout un complexe d'idées. ${ }^{77}$

La discussion sur la «loi de Say» est aujourd'hui éteinte. Rappelons d'abord brièvement que la «loi de Say» comporte un grand nombre d'énoncés différents. Le premier est celui de l'identité de JeanBaptiste Say telle qu'elle a été développée par O. Lange. ${ }^{78}$ Cette identité signifie simplement que personne ne désire jamais détenir de la monnaie pendant une durée dépassant le temps moyen entre deux transactions, la monnaie n'est qu'un moyen d'échange. Il en résulte que chaque offre d'une quantité de produit donnée constitue automatiquement une demande pour un autre marché d'un autre produit. Comme dans une

Economia, Parte Moderna, Milano, Custodi, 1804, vol. 15, p. 33-34. Il prezzo, esattamente parlando, significa la quantita di una cosa che si dà per averne un'altra (...) Presso di noi, che abbiam l'uso della merce universale, la parola prezzo significa la quantita della merce universale che si dà per un'altra merce.

76 Voir W.J. Baumol, "Jean-Baptiste Say's (at least) Eight Laws, or what JeanBaptiste Say and James Mill may have really meant”, Economica, février 1977, vol. 44, $\mathrm{N}^{\circ} 173$, pp. 145-156 et G. Becker \& W.J. Baumol, "La théorie monétaire classique: le résultat de la discusssion", Economica, novembre 1952, pp. 355-376, traduction in Textes choisis, vol I, Problèmes monétaires, Paris, Dalloz-Sirey, 1964, pp. 47-67. W.J. Baumol, Jean-Baptiste Jean Baptiste Say und der "Traité", in réédition du Traité d'Économie politique(1803), Frankfurt/Main- Düsseldorf, Verlag Wirstchaft und Finanzen $\mathrm{GmbH}, 1986$, Traduction "Jean-Baptiste Say et le Traité", par Elisabeth David avec la collaboration de A.Tiran, Working-paper, Centre A. et L.Walras, 1993.

77 W.J. Baumol, "Jean-Baptiste Say's (at least) Eight Laws, or what JeanBaptiste Say and James Mill may have really meant”, Economica, février 1977, vol. 44, $\mathrm{N}^{\circ} 173$, pp. 145-156.

78 O. Lange, "Jean-Baptiste Say's Law: a restatement and Criticism”, in Studies in Mathematical Economies and Econometrica, par O. Lange et T.O. Yntema (eds), Université de Chicago, 1942. Dans les différentes interprétations de la "loi de JeanBaptiste Say", J.A. Schumpeter distingue quatre versions différentes de "l'indentité de Jean-Baptiste Say", voir Histoire de l'Analyse économique", vol. II, op. cit., pp. 326-328. 
économie de troc l'offre crée automatiquement sa propre demande et une surproduction générale est impossible.

La seconde version de la loi de Jean-Baptiste Say est celle de l'égalité $^{79}$ cette forme de la loi admet de brèves périodes de déséquilibre durant lesquelles la demande totale pour les marchandises peut être inférieure à l'offre totale, mais cette version maintient que l'action de forces rééquilibrantes ramène vite la demande et l'offre totale à égalité. Sur la paternité de la formulation de la loi de Jean-Baptiste Say, Spengler ${ }^{80}$ a montré les antécédents chez les physiocrates et Sowell à souligné certaines formulations chez Smith. ${ }^{81}$ Thweatt ${ }^{82}$ attribue la loi à James Mill, Baumol l'attribue à Jean-Baptiste Say dans sa version d'égalité.

\subsection{La dynamique de la production chez Pietro Verri}

Pietro Verri décrit un enchaînement que l'on peut schématiser comme suit:

augmentation générale de la quantité de monnaie —— > dilatation de la sphère des besoins des citoyens_—— - >augmentation du désir de les satisfaire_— $>$ augmentation des acheteurs_—->augmentation des vendeurs_— > augmentation des producteurs.

La condition posée par Pietro Verri pour que cet accroissement de quantité de monnaie entraîne cet enchaînement vertueux est l'augmentation graduelle et l'égale répartition de la quantité de monnaie sur l'ensemble du corps social. À cette condition les prix n'augmenteront pas, ou augmenteront dans des proportions inférieures à celle de la

79 J.A. Schumpeter, Histoire de l'Analyse Économique, vol. II, pp. 322-335.

80 J. Spengler, "The physiocrats and Jean Baptiste Say's Law of markets", I, The Journal of Political Economy, septembre 1945, vol. LIII, N³, pp. 193-211 et décembre 1945, vol. LIII, pp. 317-347, voir en particulier pp. 182-184.

81 T. Sowell, La loi de Jean Baptiste Say, une analyse bistorique, 1972, Princeton University, trad. fr. ITEC,1991, Paris, pp. 10-11.

82 W.O. Thweatt, "Early Formulators of Jean-Baptiste Say's Law", Quarterly Review of Economics and Business, ${ }^{\circ}$ 19, 1979, p. 79-96, et "Baumol and J. Mill on “Say's' Law of markets”, Econometrica, n47, 1980, pp. 467-469, réédité par M. Blaug, in Jean-Baptiste Say (1776-1832), Aldershot, Elgar, 1991. Les articles de Baumol et Spengler sont également dans ce recueil. 
quantité de monnaie, et la valeur de la monnaie ne diminuera pas, ou diminuera moins que l'augmentation de sa quantité..$^{83}$

Analysant le rôle de la monnaie et son impact sur les prix lorsque sa quantité varie, Pietro Verri est amené à une formulation qui est l'exacte inversion de la loi des débouchés par Jean-Baptiste Say, celuici la formulera en s'opposant à l'analyse de Pietro Verri tout en lui empruntant la dynamique que décrit ce dernier: «J'ai dit qu'en augmentant, les achats tendent à accroître proportionnellement les vendeurs et les reproducteurs dans un Etat, car plus il y a d'acheteurs et plus croît l'utilité d'être vendeur, et plus les vendeurs augmentent et plus se multiplient d'autant les reproducteurs.» ${ }^{84}$ Pietro Verri précise: «J'ai dit que: Dans le même temps où les achats augmentent, les vendeurs et les reproducteurs tendent à augmenter proportionnellement dans un Etat parce que plus il y a d'acheteurs et plus augmente l'utilité d'être vendeur et les reproducteurs augmentent d'autant plus que les vendeurs augmentent. Car en augmentant le nombre des acheteurs, l'intérêt d'être vendeur augmente; mais en augmentant le nombre des vendeurs l'intérêt d'être acheteur n'augmente pas de la même façon». ${ }^{85}$ Pour Pietro Verri on produit et on vend des marchandisses parce qu'elles sont très recherchées et elle sont d'autant plus produites et vendues qu'elles sont plus demandées; mais des marchandises ne sont pas plus demandées parce que ceux qui les produisent et qui les vendent augmentent. Achevée cette démonstration des relations entre l'achat et la vente il s'interroge: «Mais cette théorie ne pourrait-elle pas être interprétée dans l'autre sens? Celui qui dirait que lorsque dans un Etat les vendeurs augmentent les acheteurs doivent aussi augmenter prononcerait des paroles qui ne contiennent pas une pensée réfléchie». ${ }^{86}$

Plus loin Verri précise «Chaque Nation est naturellement composée de vendeurs et d'acheteurs, chaque vendeur d'une marchandise est et

83 Verri, Meditazioni sulla economia politica, Scrittori Classici Italiani di Economia Politica, Parte Moderna, Milano, Custodi, 1804, vol. 15, pp. 50-51.

84 Verri, Meditazioni sulla economia politica, Scrittori Classici Italiani di Economia Politica, Parte Moderna, Milano, Custodi, 1804, vol. 15, p. 42.

85 Verri, Meditazioni sulla economia politica, Scrittori Classici Italiani di Economia Politica, Parte Moderna, Milano, Custodi, 1804, vol. 15, p. 42.

86 Verri, Meditazioni sulla economia politica, Scrittori Classici Italiani di Economia Politica, Parte Moderna, Milano, Custodi, 1804, vol. 15, p. 42. 
doit être acheteur des marchandises qu'il consomme, parce que sans le besoin l'bomme ne quitte pas la paresse, et ne se met pas au travail où à l'échange sinon pour chercher les moyens de se procurer les consommations propres». ${ }^{87} \mathrm{Il}$ développe son objection en traitant des rapports entre marché intérieur et marché extérieur: «chaque achat suppose une vente et chaque vente un achat, mais chaque vendeur national n'implique pas un acheteur national; mais tout au contraire l'un et l'autre peuvent être dans un pays étranger, et ainsi la somme totale des acheteurs internes peut-être supérieure ou inférieure par rapport à la somme des vendeurs intérieurs». ${ }^{88}$

\subsection{Radicalisation et rupture de Jean-Baptiste Say}

Pietro Verri perçoit bien que l'on peut inverser sa proposition et dire que «plus les producteurs augmentent avec les vendeurs et plus les acheteurs augmentent». Mais il déclare que sa formulation: «si les acheteurs augmentent les vendeurs doivent augmenter proportionnellement», doit être entendue dans un sens mais que la réciproque n'est pas vraie. Jean-Baptiste Say opère un renversement qui place la production au début de la séquence, il innove sur le plan théorique en inversant la logique initiée par Pietro Verri. On obtient alors: l'augmentation des reproducteurs crée l'augmentation des vendeurs et celle des acheteurs, car tout le monde est à la fois acheteur et vendeur. Si les vendeurs augmentent les acheteurs doivent augmenter proportionnellement. ${ }^{89} \mathrm{C}$ 'est bien comme cela qu'ensuite Jean-Baptiste Say présentera les choses, comme l'échange entre les services producteurs et les produits. Il écrit: «Leur nation leur offrira d'autant plus de débouchés, qu'elle peut payer plus de choses et elle peut payer plus de choses, à proportion de ce qu'elle en produit davantage». ${ }^{90}$ Son apport personnel se situe sur l'origine du

87 Verri, Meditazioni sulla economia politica, Scrittori Classici Italiani di Economia Politica, Parte Moderna, Milano, Custodi, 1804, vol. 15, p. 55.

88 Verri, Meditazioni sulla economia politica, in Scrittori Classici Italiani di Economia Politica, Parte Moderna, Milano, Custodi, 1804, vol. 15, p. 56.

89 Le terme de vendeur doit être ici entendu comme synonyme de producteur, quoique Pietro Verri l'utilise par moments dans une sens distintc de celui de producteur et parfois comme désignant l'ensemble des participants à l'échange quel que soit leur fonction.

90 Say, TEP, $1^{\circ}$ édition, 1803, tome 1, Paris, Crapelet, p. 154. 
déséquilibre: «En conséquence quand une nation a trop de produits dans un genre, le moyen de les écouler est d'en créer d'un autre genre». ${ }^{91} \mathrm{Le}$ point de départ se trouve aussi chez Pietro Verri, comme nous l'avons vu plus haut, dans l'idée que les besoins sont illimités. C'est de là qu'il faut partir si l'on veut saisir l'intention première de Jean-Baptiste Say qui n'est pas de formuler une loi de l'équilibre.

La première idée de Say sur le moteur de la croissance se trouve dans cette proposition simple: ayant des besoins chacun pour les satisfaire doit produire. Dans ce sens ma production est ma consommation. Dans un univers développé ou règne la division du travail ma production n'existe que par les besoins des autres, d'où la décision de produire. On suppose qu'il est absurde que des gens se mettent à produire des biens qui ne puissent pas être demandés, mais qu'au contraire ces mêmes hommes se sont assurés que la demande de biens existe avant de produire. Ce que Say veut mettre en évidence c'est le but ultime de ce circuit, la consommation, alors que tout le monde s'arrête au milieu, à l'argent.

Jean-Baptiste Say pose dés la première édition du Traité (1803) que la demande globale en valeur des biens et services est toujours égale à l'offre globale. Il affirme d'abord que la consommation n'est point une cause: «elle est un effet. Pour consommer il faut acheter; or on n'achète qu'avec ce qu'on a produit. La quantité de produits demandés est-elle donc déterminée par la quantité de produits créés? Sans aucun doute». ${ }^{92} \mathrm{Il}$ en découle que: «Chacun peut à son gré consommer ce qu'il a produit; ou bien avec son produit en acheter un autre. La demande de produits en général est donc toujours égale à la somme des produits»."

Chez Pietro Verri la production suppose au préalable une demande existante d'où le point de départ de la monnaie comme expression de cette demande. Les bienfaits qu'apporte une entrée de monnaie dans l'État si elle se fait graduellement et de façon uniformément répartie relève de cette logique. Par rapport à Pietro Verri, et c'est là sa filiation avec Smith et les physiocrates, il place au commencement de tout le processus productif l'acte de produire: l'offre. Jean-Baptiste Say s'affirme l'héritier de Pietro Verri lorsqu'il écrit quelques pages plus loin « $\mathrm{La}$

91 Say, TEP, $1^{\circ}$ édition, 1803, tome 1, Paris, Crapelet, p. 154.

92 Say, TEP, 1ère, tome 2, livre IV, p. 175.

93 Say, TEP, 1ère, tome 2, livre IV, pp. 175-176. 
première conséquence qu'on peut tirer de cette importante vérité, c'est que, dans tout ètat, plus les producteurs sont nombreux, et les productions multipliées, et plus les débouchés sont faciles, variés et vastes». ${ }^{94}$

94 Say, TEP, 2e édit., tome 1, p. 152. 